Cuad. Invest. Filol., 43 (2017), 51-71. http://doi.org/10.18172/cif.2985

\title{
ALGUNOS PROBLEMAS DE LOS SUFIJOS CON LECTURA DE CONTACTO BRUSCO
}

\author{
Antonio Fábregas \\ Universidad de Tromsø \\ antonio.fabregas@uit.no
}

RESUMEN: Este trabajo explora tres aspectos de la gramática de los sufijos asociados a lecturas de golpe -más propiamente, contacto brusco- en español: -azo, -ada y -ón. Se propone que estos sufijos pueden formar sustantivos de contacto brusco porque, por distintos motivos en cada caso, están asociados en su entrada a un núcleo que denota un límite final (]) subespecificado acerca de la dimensión sobre la que opera; la presencia de este límite relaciona sus lecturas de resultado y aumentativas con la de contacto brusco. Asimismo, este núcleo explica la interpretación de acción que reciben en ausencia de propiedades verbales, así como las lecturas que produce con ciertos adjetivos. Por último, se propone que el hecho de que la base nominal a veces se interprete como un instrumento (cabezazo 'golpe dado con la cabeza') y a veces como un locativo (morrón 'golpe dado en los morros') puede derivarse de la distinta preposición que cada afijo codifica en su entrada léxica.

PALABRAS CLAVE: derivación nominal, sufijos de golpe, aumentativos, límites intercategoriales.

\section{SOME PROBLEMS OF THE SUFFIXES WITH ABRUPT CONTACT READING}

ABSTRACT: This article explores three issues in the grammar of suffixes denoting abrupt contact in Spanish: -azo, -ada y -ón. It is proposed that these suffixes are able to denote such nouns because, in each case for a different reason, they are associated in their entry to a head denoting a final boundary (]) underspecified with respect to the dimension over which it ranges. The presence of this boundary relates their result and augmentative readings with those of abrupt contact. This head, moreover, explains the emergence of an action reading in the absence of verbal structure, as well as the readings that are produced with several adjectives. Finally, it is proposed that the fact that nominal base is sometimes interpreted as an instrument (cabezazo 'blow with the head') and sometimes as a locative (morrón 'blow on the mouth') can be derived from the different preposition that each affix codifies in their lexical entry.

KEYWORDS: noun derivation, blow suffixes, augmentatives, cross-categorial limits.

Recibido: 15/03/2016. Aceptado: 09/05/2017 


\section{Introducción: sufijos de golpe y sus problemas analíticos}

El objetivo inmediato de este artículo es el de analizar tres problemas que presentan dos sufijos que pueden formar de manera productiva sustantivos que designan, genéricamente, movimientos de contacto brusco o, como se describe en algunos trabajos, golpes (Millán Urdiales 1978; de Bruyne 1979; FailtelsonWeiser 1980; Camus Bergareche 1997; Dásková 2006; NGLE 2009, Anscombre 2013). Junto a estos dos sufijos productivos, que se ilustran en (1), estudiaremos un tercer sufijo (2) de productividad mucho menor, pero que impone a su base sistemáticamente una interpretación distinta a los otros dos, por lo que debe ser tomado en consideración para garantizar la solidez de las generalizaciones estructurales que haremos sobre los otros dos.

(1) a. cabez-azo

b. pedr-ada

(2) bofet-ón

Metodológicamente, en este trabajo no tenemos en consideración las voces en que la base sea ya un sustantivo (torta > tortazo) o verbo (patear > pateada, punzar > punzada, latiguear > latigueada, volcar > volquetazo, revolcar $>$ revolcón, empujar > empujón) que designa por sí mismo un movimiento de contacto brusco. En tales casos cabe pensar que el sufijo tiene su significado habitual como aumentativo (pelm-azo) o sufijo de acción (roci-ada, remoj-ón) y por tanto no es responsable de esta contribución semántica.

Antes de introducir los tres problemas analíticos en los que nos concentraremos en este trabajo, describamos sucintamente las propiedades de estas formaciones.

El sufijo -azo es el más productivo en la formación de nombres de golpe a partir de sustantivos (3) y, ocasionalmente, verbos (4).

(3) a. cabezazo, cornazo, puñetazo, rodillazo, lengüetazo, aguijonazo

b. salivazo, guantazo, capotazo, sombrerazo, zapatazo, zapatillazo

c. cañonazo, bombazo, sablazo, pistoletazo, escopetazo, metrallazo, trabucazo, flechazo, morterazo, hachazo

d. balonazo, portazo, cañazo, tijeretazo, cerrojazo, escobazo, chispazo, almo hedazo, martillazo, jeringazo

(4) arañazo, frenazo, patinazo 
Como se ve, las bases pueden referirse a partes del cuerpo de las personas o los animales (3a); a objetos estrechamente relacionados con estos, como las emisiones corporales y las prendas de vestir (3b); a armas (3c) o a muchos otros objetos que pueden usarse como instrumentos. Dos notas son importantes con respecto a la interpretación de estos sustantivos: primero, la noción de golpe no está siempre presente, pero sí la de contacto brusco. Un bombazo no es un golpe dado con una bomba, pero sí una deflagración brusca e intensa; un cerrojazo tampoco es un golpe dado con un cerrojo, pero sí un cierre brusco que emplea un cerrojo. En segundo lugar, la base nominal en (3) siempre se interpreta como el instrumento empleado para causar el contacto brusco. Un cabezazo, por ejemplo, es un golpe dado con la cabeza, pero la persona que recibe el golpe puede sentir el impacto en cualquier parte de su cuerpo.

No sucede lo mismo en unos pocos derivados con este sufijo, ilustrados en (5).

(5) cogotazo, espaldarazo

Un cogotazo no es un golpe dado con el cogote, sino en el cogote de la otra persona. El instrumento que se use para infligir el golpe es irrelevante para la denotación de la palabra; igualmente, en su sentido no figurado, un espaldarazo es un empujón dado a alguien en la espalda, no con la espalda.

El segundo sufijo productivo que estudiamos aquí es -ada, con una productividad algo menor al anterior, pero que también forma nombres de contacto brusco a partir de sustantivos.

(6) a. cornada, uñada, dentellada, patada

b. puñalada, estocada, lancetada, ballestada, perdigonada, espolada, porrada, navajada

c. pedrada, tijeretada, azadada

Tenemos aquí ya algunas categorías conocidas: partes del cuerpo de personas y animales (6a), armas (6b), junto a otros objetos que pueden usarse como instrumentos (6c). En estas palabras, de nuevo la base nominal es el instrumento usado para establecer el contacto: una uñada es un surco hecho con las uñas, no una herida infligida en las uñas. Con una productividad mucho menor, sin embargo, hay algunos derivados donde la base designa el lugar en que se produce el contacto.

(7) carrillada, gaznatada 
Una carrillada no es un golpe dado con el carrillo, sino en el carrillo. El tercer sufijo que estudiaremos aquí, -ón, no produce productivamente nombres de contacto brusco, pero es relevante porque sistemáticamente todas las voces que se forman a partir de él dentro de esta clase semántica tienen la propiedad de que la base siempre se refiere al lugar en que se produce el contacto; la única excepción parcial es molondrón, de molondra ('cabeza'), que según los diccionarios puede ser también un golpe dado con la cabeza.

(8) capón, pescozón, morrón, molondrón

Partiendo de estos datos, queremos destacar tres problemas analíticos que se asocian a ellos:

i) ¿Por qué estos afijos y no otros?

Al lector atento no se le ha escapado que dos de estos sufijos son más productivos como aumentativos (-azo, -ón) mientras que el tercero está claramente emparentado con una forma participial femenina. ¿Es esto una coincidencia o bien hay alguna razón profunda para que suceda? En este trabajo, como es habitual en el método científico, no creeremos en coincidencias y trataremos de proponer una explicación plausible a la pregunta de por qué los dos sufijos más productivos en español para producir nombres de contacto brusco se relacionan con participios y aumentativos. Pasemos al segundo problema.

ii) ¿De dónde surge la lectura de acción brusca sin base verbal?

El contacto brusco es un subtipo de acción brusca. La interpretación conceptual de los sustantivos que estudiamos aquí es claramente una en la que se describe un movimiento, pero ni la base ni el propio sufijo parecen tener independientemente un significado de acción. ¿De dónde surge, entonces, esta interpretación? ¿Hay que resignarse a que el significado de contacto brusco esté presente como parte de la entrada idiosincrásica de la palabra completa, por muy productivo que sea el proceso? Dado cualquier nombre de objeto que pueda ser empleado para golpear a alguien, los hablantes pueden producir creativamente una formación en -azo que describa el golpe. Dependiendo de qué objeto de los que tiene alguien en su escritorio sea el que nos lance, podremos decir cualquiera de las secuencias no asentadas en la lengua que se presentan en (9) y nuestro interlocutor entenderá perfectamente qué es lo que sucedió: 
(9) Pedro me propinó un \{librazo / grapadorazo / ratonazo / pisapapelazo / agendazo / monitorazo \} tremendo.

iii) ¿De qué depende que a veces la base sea el lugar donde se golpea y a veces el objeto con el que se golpea?

La última pregunta es qué es lo que condiciona la interpretación de la base, una vez que está asentada la semántica de contacto brusco, que parece independiente de esta propiedad en la medida en que se aceptan dos lecturas alternativas: instrumento o lugar. Vemos que los sufijos productivos tienen tendencia a que la interpretación que emerja sea la instrumental ('contacto brusco usando N'), y que en mucha menor medida, pero de forma casi exclusiva con -ón, surja una locativa ('contacto brusco en N'). De nuevo se produce aquí el mismo problema: ¿tratamos la alternancia como un caso de idiosincrasia o puede encontrarse alguna causa estructural que fuerce una u otra interpretación a partir de reglas?

El trabajo se estructura de la siguiente manera. La sección §2 se dedica a la primera pregunta, la de proponer una explicación a por qué son precisamente aumentativos y participios los que deberíamos esperar como afijos de contacto brusco si una lengua carece de formas especializadas. §3 estudia la segunda pregunta, de dónde surge la semántica de acción, mientras que $\S 4$ estudia la alternancia entre instrumento y lugar. Por fin, $§ 5$ presenta algunas conclusiones.

\section{Por qué estos afijos}

La primera cuestión es qué motivo hay para que sean precisamente estos y no otros los sufijos que han desarrollado significados de contacto brusco. Si atendemos al primero de ellos, -azo, es bien sabido que su uso fundamental es como aumentativo (10a), sobre todo con sustantivos o adjetivos convertidos a sustantivos (Lázaro Mora 1999, NGLE 2009: §9.7), aunque se documenta al menos en áreas andinas una extensión de este uso como variante del grado superlativo en adjetivos (10b; cf. Cerrón Palomino 2015).

$$
\begin{aligned}
& \text { (10) a. perr-azo, buen-azo } \\
& \text { b. mal-azo }
\end{aligned}
$$

En cuanto al sufijo -ón, su valor principal es el de aumentativo en combinación con sustantivos y adjetivos sustantivados (11a), si bien tiene otros usos productivos en los que también agrega la idea de exceso, como la formación de 
sustantivos y adjetivos deverbales de valor agentivo y frecuentativo: (11b) indica una persona que se caracteriza por abusar mucho, muy frecuentemente o muy intensamente. También se observa, ocasionalmente, un uso de este mismo sufijo en la formación de nominalizaciones deverbales, siempre indicando un cambio o movimiento brusco e intenso (11c).

$$
\begin{aligned}
& \text { (11) a. notici-ón, grandull-ón } \\
& \text { b. abus-ón } \\
& \text { c. calent-ón }
\end{aligned}
$$

Otras formaciones parecidas a (11c), todas ellas con el matiz de 'brusquedad' o ‘intensidad' son remojón, empujón y revolcón. No obstante, es claro que el uso más productivo de este sufijo es el de aumentativo.

En cuanto a -ada, su parentesco al menos histórico con las formaciones participiales es evidente. Es bien sabido que hay ciertas nominalizaciones participiales (12a), y aquellas que tienen género femenino tienden a expresar acciones delimitadas, rápidas y enérgicas (Fábregas 2010): darle a algo una \{chupada / limpiada / pasada / rociada / frotada\} rápida. Lo interesante de estas formaciones es que suelen expresar acciones acotadas, incluso cuando sus bases verbales no sean necesariamente télicas, como es el caso de chupar, donde es fácil contruir el predicado como atélico (chupar un palo durante horas). Junto a esto, como es bien sabido también, los participios productivamente expresan el resultado alcanzado tras la culminación de un evento (12b, 12c).

$$
\begin{aligned}
& \text { (12) a. chupa-da } \\
& \text { b. cansa-do } \\
& \text { c. limpia-do }
\end{aligned}
$$

La pregunta es cómo se pueden relacionar estas nociones con la de movimiento brusco. Para ello partiremos de la teoría aspectual de Piñón (1997). En su análisis de la clase aspectual de los logros, Piñón propone una ontología con dos clases de elementos: límites (13a, 13b) y cuerpos (13c).

(13) a. [
b. ]
c. -------- 
Dentro de un verbo, el cuerpo corresponde a la parte durativa de la eventualidad, tanto si es dinámica (correr, volar) como si es estática (saber, merecer). Los límites corresponden, respectivamente, al inicio de la eventualidad (13a), que marca el punto en el que se pasa de una situación en que la eventualidad no sucede a una en que sí, y el final de la eventualidad o culminación (13b). No todos los predicados contienen los tres elementos, y es una cuestión empírica qué verbos o morfemas denotan (por oposición a implicar o presuponer) cada uno de estos elementos ontológicos. Una consecuencia interesante de la propuesta de Piñón, que trata los límites como entidades separadas de los cuerpos que acotan, es que se abre la posibilidad de que un verbo denote solamente un límite, sin cuerpo. Esta es su propuesta para los logros, que para él tienen carácter puntual precisamente porque denotan límites sin cuerpo; véase también Marín y McNally (2011).

Como se puede deducir, esta ontología de límites (puntos) y cuerpos (intervalos) es en principio independiente de la clase léxica de las formas a las que se asocie cada primitivo. La noción de límite es en principio traducible más allá de los verbos, donde designa distintas clases de aspecto, para capturar otras nociones propias de distintas categorías, como el contraste entre masa / contable en los sustantivos o la delimitación de una escala adjetival.

La idea en el fondo es relativamente clásica: Ross (1973), Mourelatos (1978), Bach (1986) y Jackendoff (1997), entre otros, ya argumentaron que había motivos para proponer que debe existir un vocabulario de primitivos 'subcategoriales' que expresan nociones de significado generalizadas que comparten distintas categorías. La razón era doble. Por un lado, se hace necesario explicar que existan casos de quasi-concordancia entre la delimitación de una categoría y la delimitación de otra con la que forma sintagma (comer una manzana / comer arroz). Por otro lado, son innegables los casos en que palabras que en principio deberían pertenecer a categorías distintas presentan propiedades en común, o de transición entre esas dos clases y, a veces, una tercera (Bosque 1989).

La propuesta explícita de que las nociones de límite y cuerpo pueden extenderse como rasgos subcategoriales más allá de los verbos se ha desarrollado en Fábregas y Marín (2014) para las escalas adjetivales del español, y en Berro (2015) para la relación entre aspecto, nominalización y locación preposicional en vasco.

Pues bien, partiendo de esta base teórica, nuestra propuesta es que la denotación de los sufijos -azo, -ón y -ada es la de (14): un límite final; más específicamente, trato esta denotación de límite final como un operador cuyo abarque está subespecificado acerca del dominio sobre el que actúa. En principio, puede aplicarse a un dominio de escalas de grado (cuando es adjetivo), uno de canti- 
dades o partes mereológicas (cuando es sustantivo), o incluso eventualidades (cuando es verbo).

Lo que sucede es que esta semántica, argumentaremos, es común al aumentativo, al resultado y a la lectura de movimiento brusco, lo cual explica que sean precisamente los sufijos que denotan esto los que pueden ser reutilizados para expresar golpes.

Repasemos ahora cómo esta denotación tan simple da la semántica relevante. (14) es un límite final, que por lo tanto cierra, dando el valor máximo, la serie que forma un conjunto ordenado de puntos. Es la naturaleza dimensional de este conjunto de puntos lo que nos da la diferencia entre las lecturas.

Empecemos con la lectura aumentativa, que es la más productiva para -azo y -ón. Lo que denota un aumentativo es que cierta cantidad, cierta magnitud o cierta propiedad aparecen en un grado excesivo que se sitúa en el límite superior de lo esperable para esa clase de objetos. Si alguien es un buenazo, es que está en el límite máximo esperable para un ser humano en la propiedad de 'ser bueno', igual que si alguien obtiene un sueldazo es que la cantidad monetaria que recibe se encuentra en el límite superior de las cantidades que el hablante conoce que se aplican a los sueldos. Esta lectura puede expresarse si dentro de la estructura de la palabra el límite inicial proyecta como un núcleo cuyo complemento es un núcleo de cuantificación.

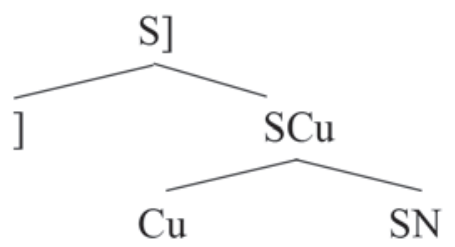

Si se aplica a un adjetivo, proponemos que el mismo límite abarca sobre un núcleo de escala; tampoco son sorprendentes desde esta perspectiva los datos de Cerrón Palomino (2015) en los que -azo ha pasado a expresar superlativo: de nuevo estamos en el límite superior de una serie ordenada de puntos, en este caso valores de grado.

Tampoco es difícil entender la relación entre este primitivo y las nociones de acción culminada y estado resultante asociadas a la forma participial. Si el 
mismo límite final opera sobre una noción de aspecto, obtendremos la lectura de que tenemos un aspecto acotado; para poder establecer la noción de resultado que sigue a esa culminación -por oposición a un estado no resultante- tendremos que construir un estado que siga a ese límite final, con esquema ']-------', por lo que el límite final debe estar presente.

Nuestra propuesta es que este límite ']' es el mismo primitivo que se toma como básico para denotar contacto con una entidad: aplicado a ciertas nociones que definen a la base como instrumentos o lugares, como veremos en §4, lo que esperamos es que este límite se interprete como la culminación del uso instrumental del objeto (por tanto, estableciendo contacto con una superficie) o, cuando se aplica a una región espacial denotada por la base, contacto con el límite final de un objeto, es decir, con el perímetro de esa región.

En este sentido la noción de contacto que se asocia a este límite hace que ']' se aproxime por sus propiedades a las preposiciones llamadas de coincidencia terminal (Hale 1986, Hale y Keyser 2002, Mateu 2002). Estas preposiciones expresan un contacto periférico entre dos objetos, frente a las de concidencia central, que denotan la inclusión de un objeto en otro y se asocian a lecturas estáticas en que dicha relación permanece estable durante el intervalo en que tiene lugar una eventualidad.

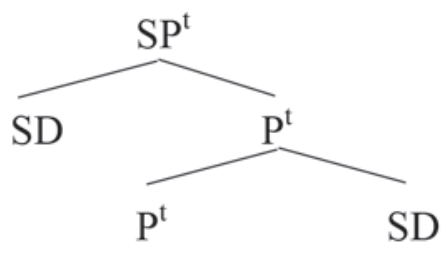

En (16), siguiendo el principio propuesto en Hale y Keyser (2002) de que un núcleo relacional debe contener dos argumentos, hay dos sintagmas determinantes introducidos dentro de la proyección del núcleo. Estos denotan, respectivamente, cada una de las dos entidades que entran en contacto. La conexión, pues, del límite final con las preposiciones de contacto terminal es muy estrecha.

¿Por qué debe ser brusco este contacto? La explicación es que la denotación de estos sufijos expresa el límite final sin denotar el cuerpo que precede a ese límite final. En nuestro caso, ese cuerpo sería el movimiento que culmina en el contacto, pero al no estar presente, el único elemento que se mantiene es el mismo contacto en sí, que es puntual y no durativo. Por esta razón, intuitivamente, estos sustantivos de 
golpe se interpretan como contactos súbitos, brusco, a veces inesperados, en los que se no se denota ninguna parte de la acción que conduce a producirlos. Pero pasemos ahora a la segunda pregunta, ya que introducirá elementos que pueden iluminar más la cuestión del tipo de acción que se expresa con estos verbos.

\section{De qué depende la lectura de acción brusca}

Para afrontar esta pregunta resulta útil comparar las formaciones de contacto brusco con otros casos donde tenemos eventos expresados en sustantivos. Las dos clases mayores a las que hay que oponer estas formaciones (como en 17c) son los nombres de evento derivados de verbos (17a) y los nombres eventivos no derivados de verbos (17b). Como es bien sabido, Grimshaw (1990) llamó a los primeros 'nombres de evento complejo' y a los segundos, 'nombres de evento simple'.
(17) a. apaleamiento
b. paliza
c. puñetazo

Existe un acuerdo general en que los nombres de evento complejo heredan la estructura argumental del verbo usado como su base y los de evento simple, como otros sustantivos, carecen de estructura argumental propia o heredada. Esto se manifiesta, entre otras cosas, en la distinta aceptabilidad de sintagmas preposicionales que usen preposiciones distintas a de y con en interpretación de argumento interno. (18a) muestra que los nombres de evento complejo lo permiten; como se ve en (18b), los de evento simple solo lo hacen muy marginalmente, y nuestros sustantivos de contacto brusco los rechazan claramente (18c).

(18) a. El apaleamiento a los detenidos fue breve.

b. ??La paliza a los detenidos fue breve.

c. *El puñetazo a Juan fue breve.

Esto ha recibido habitualmente la explicación de que ciertas preposiciones asociadas a la proyección de argumentos solo están legitimadas en el interior de grupos verbales (Fábregas 2014), de donde se sigue -como por otro lado muestra composicionalmente la estructura morfológica- que solo (18a) contiene un auténtico sintagma verbal. De aquí derivamos la siguiente consecuencia para nuestros nombres de contacto brusco: 
i) La lectura de contacto brusco no se debe a la presencia de un núcleo verbal que introduzca estructura argumental

Pasemos a una segunda propiedad. Los nombres de evento complejo, por la misma presencia de estructura verbal que permitía legitimar argumentos, aceptan modificadores de aspecto y tiempo (19a). Los de evento simple los rechazan taxativamente (19b), y nuestros sustantivos se comportan también como ellos (19c). Probamos tanto con un modificador delimitador como con uno atélico, para evitar que la incompatibilidad se deba a un choque entre las supuestas propiedades aspectuales del sustantivo y la presuposición que hace el modificador.

(19) a. su apaleamiento durante horas

b. *su paliza durante horas

c. *su puñetazo \{en un segundo / durante horas

Consecuentemente, solo el primero tiene proyecciones aspectuales modificables, de donde seguimos una segunda conclusión para nuestros datos:

ii) Los nombres de contacto brusco carecen de proyecciones (sub)eventivas aspectuales.

Pasemos a una tercera propiedad en la que coinciden tanto los nombres de evento complejo como los de evento simple: la capacidad de estar seleccionados por predicados que imponen una lectura de evento a sus argumentos, como sucede con el sujeto de tener lugar (cf. *La mesa tuvo lugar ayer, que es agramatical incluso en la lectura plausible de 'La mesa fue construida ayer'). Los nombres de contacto brusco son los únicos del grupo que rechazan esta construcción (20c).

(20) a. Su apaleamiento tuvo lugar ayer.

b. La paliza tuvo lugar ayer.

c. *El puñetazo tuvo lugar ayer.

La interpretación de este hecho es algo más polémica; una explicación más bien habitual de (20a) y (20b) es que la selección que hace tener lugar de su argumento es conceptual: lo que es relevante para este predicado es que el significado de la palabra sea compatible con una interpretación eventiva, y es indiferente a su esa interpretación se sigue de la estructura porque contenga proyecciones verbales o debe ser parte de la información semántica conceptual del sustantivo cuando no es derivado, como es plausiblemente el caso de paliza (o guerra, fiesta, terremoto, conferencia, etc.): un sustantivo no derivado que por motivos de su 
entrada léxica denota un tipo de evento, igual que miedo denota un tipo de estado y lápiz denota un tipo de objeto físico. Si esta explicación está bien encaminada, se sigue la tercera consecuencia para nuestros datos:

iii) Los nombres de contacto brusco no denotan propiamente eventos tampoco a un nivel conceptual.

Para aclarar mejor qué significa esto, pasemos, por último, a la interpretación que reciben ciertos adjetivos de velocidad en combinación con nuestros sustantivos. Los adjetivos de velocidad son interesantes desde esta perspectiva, porque la velocidad es una propiedad de los eventos dinámicos (cf. *Sabía inglés rápidamente) y por lo tanto estos adjetivos no pueden incidir sobre sintagmas que expresen proposiciones (*Que Juan saliera de su habitación fue rápido) u objetos físicos (*un árbol rápido, frente a una lectura rápida), salvo lecturas sincategoremáticas (un coche rápido, comida rápida). Pues bien, como se ve en (21), de forma no sorprendente para las dos primeras clases pero aparentemente contradictoria para la tercera clase, los tres grupos de sustantivos aceptan estos adjetivos.

(21) a. Su apaleamiento fue rápido.

b. La paliza fue rápida.

c. El puñetazo fue rápido.

Es importante, sin embargo, atender a la interpretación que se produce con estos sustantivos, que no es igual. Tanto (21a) como (21b) hablan de la velocidad con la que se desarrolla el evento, lo cual afecta a la distancia que media entre el principio y el final de la acción: una paliza rápida dura menos que una paliza lenta. En cambio, un puñetazo rápido y uno lento duran exactamente lo mismo, porque lo que modifica el adjetivo en este caso es la distancia que media entre el momento en que se produce el puñetazo (es decir, el impacto contra el otro cuerpo) y un punto arbitrario anterior en el tiempo. Es, en sentido más intuitivo, el movimiento del brazo que antecede al puñetazo lo que puede ser más o menos rápido.

Una prueba de que esta es la interpretación correcta de (21) es que (21a) y (21b) pueden, ambos, sustituir el adjetivo de velocidad por uno de duración que expresamente cuantifica la distancia entre inicio y final de la acción: \{Su apaleamiento / La paliza\} fue breve. No sucede lo mismo con el tercero, precisamente porque el sustantivo, como hemos concluido de las observaciones anteriores, carece de un componente de acción a todos los niveles: * El puñetazo fue breve. 
Todas estas propiedades son derivables si la lectura de contacto brusco se debe exclusivamente a la presencia de ']' como un elemento expresado como parte del sufijo: no hay eventividad expresada en forma de estructura verbal; no hay una semántica conceptual que defina el sentido de evento, al contrario que en sustantivos como guerra o paliza. La interpretación de que hablamos de una acción puede deberse a un efecto pragmático, pero semánticamente lo único que se denota es la existencia de un límite final, interpretado contextualmente como el contacto entre dos objetos.

La semántica de ']' también explica la interpretación de los adjetivos de velocidad: ya que no se denota un cuerpo, sino solo un límite, no existe ningún intervalo que pueda medirse, al contrario de lo que sucede con los otros tipos de sustantivo considerados aquí. Que interpretemos que lo rápido es el momento que precede al puñetazo (la acción de desplazar el brazo) se sigue de que el sufijo solo aporte el valor del límite (cuando se produce el impacto), por lo que la interpretación se acomoda para expresar un intervalo arbitrario que precede al límite final.

Por este motivo, el contacto expresado con estos afijos debe ser brusco: no se denota en su estructura semántica interna intervalo alguno, y concretamente no se denota el proceso que precede al impacto. Así, la lectura natural que surge es la de que se produce un impacto de forma brusca, y sin preparación previa.

\section{La alternancia entre instrumento y lugar}

Hemos visto que, aunque las formaciones productivas siguen siempre el esquema en que la base nominal se interpreta como el instrumento que produce el contacto brusco, hay algunas formas en que se emplea la lectura locativa, donde la base expresa el lugar en que se produce el contacto. Estas formaciones son poco productivas: el puñado de formaciones con -ón, y unos pocos derivados con -azo y -ada.

La pregunta en este punto es cómo dar cuenta de estos casos locativos. Este es un tema clásico en los estudios de morfología: cuando hay una lectura aparentemente impredecible o arbitraria, ¿debe restringirse la interpretación mediante propiedades de la base -algo que compartan todos los nombres que dan lectura de lugar-, mediante las propiedades del afijo -polisémico entre instrumento y lugar- o para toda la palabra completa -listando las formaciones locativas como excepcionales-? 
A un nivel puramente empírico, la generalización que queremos expresar (y, en el mejor de los casos, derivar) es que el sufijo -azo y el sufijo -ada solo significan 'lugar en que se produce el contacto' con unas pocas bases, mientras que -ón siempre significa 'lugar', aunque sea poco productivo. Hay dos vías concebibles para tratar de capturar esta generalización:

(a) asignar el significado de instrumento como parte de la entrada conceptual del sufijo y listar idiosincrásicamente la forma locativa para -azo, haciendo lo contrario para -ón

(b) relacionar léxicamente las bases irregulares con una proyección o propiedad adicional que invierte la interpretación del sufijo, desde instrumental (que sería la versión no marcada) hasta locativa

La primera solución funcionaría de la siguiente manera. En por ejemplo cabezazo (22), donde tenemos la lectura productiva de -azo como instrumento para realizar el golpe, no habría necesidad más que de combinar la entrada de -azo con la de cabez(a).

(22) a. -az(o) <----> contacto con N

b. cabez(a) <----> parte superior del cuerpo

Ahora bien, en una palabra como espaldarazo, que es más bien un golpe dado en la espalda, sería necesario listar las palabras como excepciones, lo cual nos lleva a la siguiente entrada para -azo, con el significado instrumental como el valor por defecto y el valor locativo solo presente con algunas bases léxicamente especificadas:

(23) - az(o) <----> si $\mathrm{N}=$ espald(a)... / contacto en $\mathrm{N}$ Resto / contacto con $\mathrm{N}$

Tenemos también caso de voces en las que coexisten los dos significados. Esto sucede con molondrón, que es tanto el golpe dado con la cabeza como el golpe dado en la cabeza. En tal caso, podemos suponer que -ón tiene una entrada léxica que indica 'contacto en', y que esta entrada es la que se activa para el valor locativo de molondrón (24a). La lectura instrumental en el caso de este sufijo se seguiría de una entrada idiomática para esta palabra, como formación completa (24b).

(24) a. -ón <-----> contacto en

b. [[molondr]ón] <----> golpe dado con la cabeza 
Esta solución es muy atractiva, y es prácticamente imposible descartarla de forma tajante, toda vez que procedimientos de listado léxico como estos son independientemente necesarios para muchas otras formas. Por ejemplo, -eza es productivo formando nominalizaciones de cualidad (belleza, tristeza, grandeza...), pero en limpieza puede usarse para denotar eventos (La limpieza de los baños tiene lugar cada día a las ocho); no parece sencillo escapar a una solución como la de (23) para esta formación, donde se liste que el sufijo significa 'evento relacionado con A' con la base limpi(o). Asimismo, situaciones como las de (24) se dan habitualmente: pensemos en el significado composicional de contable 'que se puede contar', y su uso técnico impredecible generalizado en lingüística, 'clase de sustantivos que denotan entidades acotadas y enumerables'. Podría ser que esto fuera todo lo que hay que decir sobre la alternancia. Sin embargo, antes de acabar este trabajo queremos explorar brevemente la segunda solución, y ver hasta dónde nos lleva.

Si pensamos en términos estructurales, y nos planteamos la naturaleza de las relaciones instrumentales y locativas, la primera observación es que tanto ser instrumental como ser locativo son nociones que en español se expresan preposicionalmente (con un martillo, en la cocina). Siguiendo a Fábregas y Varela (2006) y Oltra-Massuet y Castroviejo (2014) vamos a dar por hecho que existen preposiciones especializadas para estas nociones, al menos en lo que toca a la dimensión de significado que especifican -locación, instrumentalidad, temporalidad, etc.- y por lo tanto actúen como funciones que convierten su complemento nominal en, respectivamente, una región espacial (véase Wunderlich 1991), un instrumento, una manera (Alexeyenko 2015), etc. Posiblemente la estructura abstracta que puede encabezar una preposición sea igual en todos los casos (Svenonius 2010), pero nos abstraeremos de estos detalles, que resultan ortogonales para nuestro propósito. Lo que nos resulta crucial es que la estructura que proponemos para estas formaciones es la de (25):

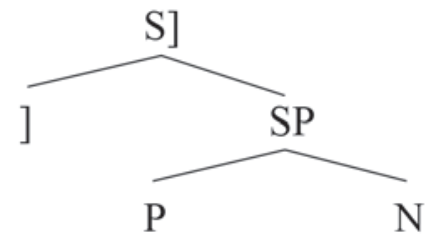

La diferencia entre la lectura instrumental y la lectura locativa la dará el tipo de preposición. El límite final se interpreta forzosamente como contacto con un objeto en la configuración de (25), donde lleva como complemento un sustantivo con marca preposicional. 
La estructura, y concretamente el orden en que se introducen los elementos que forman el sustantivo de contacto brusco, está forzada, así, por la interpretación. La preposición no puede dominar S], porque lo que queremos expresar no es que el límite de contacto sea instrumental o locativo, sino que la base debe interpretarse como un instrumento asociado a ese límite de contacto, o como un lugar que define el área de ese límite de contacto. Por tanto la estructura tendrá que ser la que se muestra a continuación, una vez eliminado el SQu de -azo y el SAsp de -ada, que definen solo los sufijos como aumentativos y aspectuales.

Podemos proponer que a partir de esta estructura el material sintáctico que se asocia a los sufijos -azo y -ada de hecho lexicalizan el conjunto formado por ] y Pinstr (véase Ramchand 2008 para el procedimiento técnico que expresa esta relación).

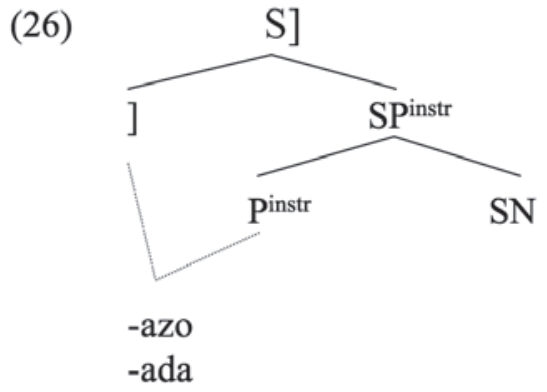

Esto captura el hecho de que cuando forman derivados sustantivos, el sufijo expresa una noción de movimiento brusco que se asocia por defecto a una lectura instrumental. En cambio, -ón tendría la estructura asociada que se muestra a continuación, donde expresa la relación locativa.

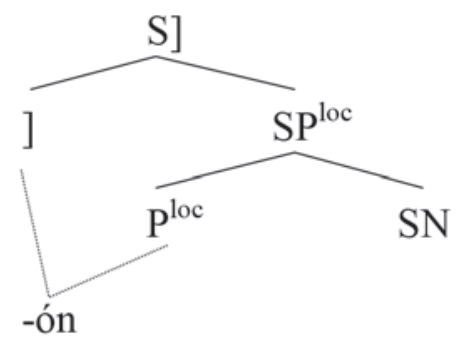

Siguiendo con este sistema en el que lo crucial es la estructura interna de la formación derivada, para dar cuenta de los casos excepcionales en que la base se interpreta en la lectura marcada para cada afijo, la principal vía de análisis es 
forzar que la preposición que aparezca con esas bases en particular sea distinta a la que lexicaliza el sufijo. Esto implicaría como un primer intento darle la siguiente estructura a un nombre como espaldarazo:

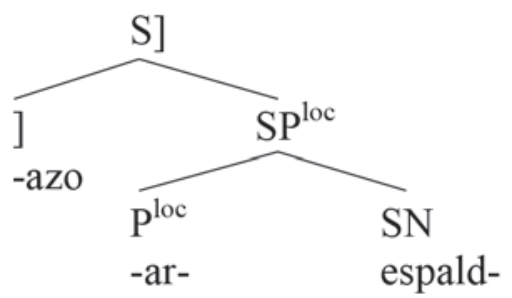

Como se ve en (28), una posibilidad sugerente sería que en este contexto el interfijo -ar- lexicaliza Ploc, la preposición adicional que no está cubierta en la entrada léxica del sufijo, ya que -podría razonarse- ni el sustantivo por sí solo ni el sufijo pueden lexicalizar esta preposición de lugar. Sin embargo, espaldarazo es el único caso de palabra con interfijo dentro de nuestra serie con interpretación marcada de la base; cabría proponer que los demás términos semánticamente irregulares contienen la misma preposición, pero que contextualmente se realiza como cero, aunque esta propuesta tendría poca fundamentación empírica.

Lo que propondremos es lo siguiente: los sustantivos que actúan como bases irregulares en estos casos tienen entradas léxicas como las de (29), es decir, ya expresan como parte de su entrada la preposición que les da la lectura irregular:

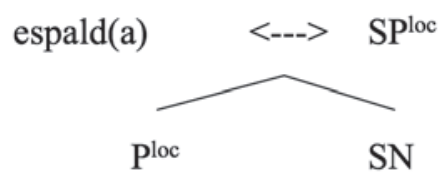

¿Por qué entonces no aparecen como nombres escuetos cuando son modificadores de lugar? La respuesta está en que esa entrada léxica solo permite una materialización cero de la preposición si P está inmediatamente adyacente al SN, formando un constituyente en exclusión del resto de elementos del sintagma. $\mathrm{O}$, dicho de otro modo, esta entrada solo predice que si el sustantivo aparece sin determinantes o flexión de número, podrá asociarse a una lectura de lugar. Cuando tenemos, en cambio, un modificador de lugar que contenga este sintagma nominal completamente expandido (e.g., en la espalda), sin embargo, habrá al menos un SNúmero entre los dos elementos, por lo que $\mathrm{P}$ y $\mathrm{N}$ no son adyacentes y garantizamos que en esa construcción debe materializarse la preposición: 
(29)

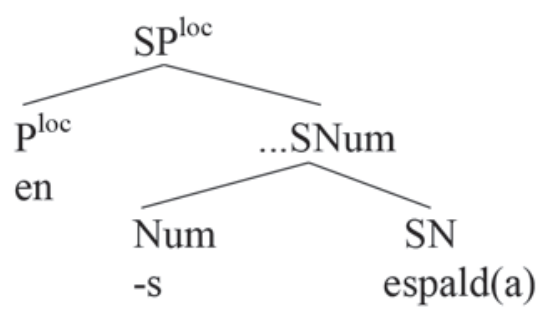

Esto bloquea la manifestación cero de la preposición, porque en ese caso la preposición y el SN no forman un constituyente en exclusión de (al menos) SNum.

Hablemos, por último, del caso de molondrón, que es la única voz derivada con -ón que admite (junto a la lectura locativa, regular con el sufijo) una interpretación instrumental de la base. No hemos conseguido encontrar datos suficientes para determinar si los dos sentidos ('golpe con la cabeza' y 'golpe en la cabeza') se dan en hablantes distintos (o en registros distintos del mismo hablante) o conviven en el mismo código para un solo hablante. De ser lo primero, la diferencia entre las dos lecturas se debería exclusivamente a si el sustantivo molondra tiene en la variedad relevante la posibilidad de lexicalizar una preposición instrumental por sí solo, de la misma manera en que espalda lexicaliza una de lugar, o no:

(30) a. molondrón, 'golpe en la cabeza'

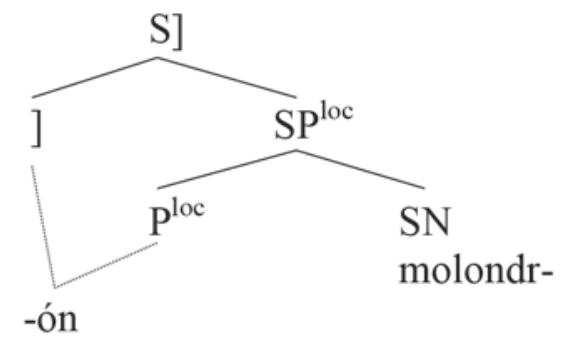

(30) b. molondrón, 'golpe con la cabeza'

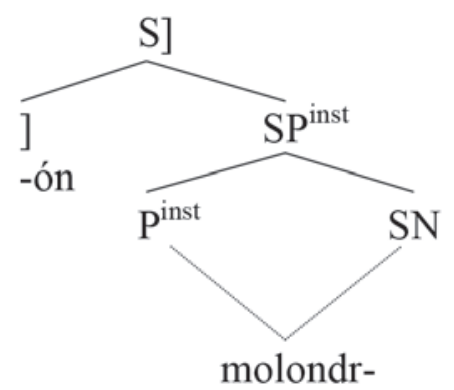


En cambio, si el mismo hablante admite las dos interpretaciones, la forma más directa de capturar este hecho es dejar que la estructura sintáctica esté subespecificada, mediante una P que no lleve información sintáctica sobre el papel temático que asigna a la base o la función semántica que define; esto permite que se interprete solamente que la base tiene que ser una entidad relacionada con el movimiento brusco, pero sin especificar si es el lugar en que se entra en contacto o el instrumento usado.

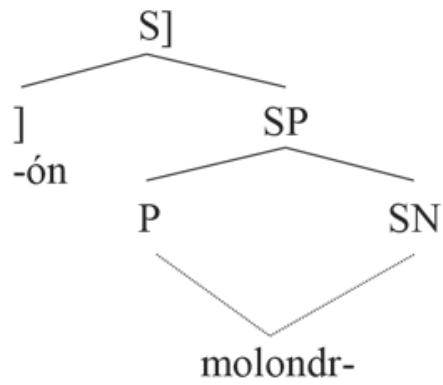

Dejamos, pues, esta última parte como un problema abierto para investigación posterior.

\section{Conclusiones}

En este trabajo hemos explorado tres cuestiones relacionadas con los nombres que denotan contacto brusco. Hemos argumentado que los sufijos que los producen son aumentativos o resultativos porque contienen en su denotación un rasgo ']' que se puede reutilizar para manifestar la noción de contacto. La conclusión general de esta parte de nuestra investigación es que la existencia de estos derivados constituye evidencia adicional de que es necesario distinguir un vocabulario de primitivos subcategoriales que expresen nociones de límite superior e inferior sin estar directamente asociadas a adjetivos, sustantivos o verbos.

El segundo aspecto estudiado es la relación que tienen estos sustantivos con la noción general de evento, y ahí hemos argumentado que no es necesario dadas las propiedades empíricas que exhiben- proponer primitivos estrictamente aspectuales o verbales en su estructura interna, tal y como la forma superficial de estos elementos sugiere. La noción acategorial de límite final es suficiente para expresar la idea de movimiento brusco, y da cuenta además sin presuposiciones 
adicionales de la interpretación que reciben los adjetivos de velocidad con estos sustantivos.

Finalmente, aunque de forma más preliminar, hemos propuesto que tal vez no es necesario listar como formas idiosincrásicas las palabras completas en las que se produce una interpretación semántica marcada para el sufijo: permitir que la base lexicalice en esa configuración una preposición marcada puede bastar.

\section{Bibliografía}

ALEXEYENKO, S. (2015). The syntax and semantics of manner modification: adjectives and adverbs, Tesis doctoral. Osnabrück: University of Osnabrük. ANSCOMBRE, J.-C. (2013). "El sufijo -ón en español contemporáneo: morfología y prototipos”, Oralia 16: 11-32.

BACH, E. (1986). "The Algebra of Events”, Linguistics and Philosophy 9: 5-16.

BERRO, A. (2015). Breaking verbs. From event structure to syntactic categories in Basque, Tesis Doctoral. Vitoria-Bordeaux: UPV/EHU-Université Bordeaux Montaigne (UBM).

BOSQUE, I. (1989). Las categorías gramaticales. Madrid: Síntesis.

CAMUS BERGARECHE, B. (1997). "Sufijos apreciativos con derivados verbales en español”, Revista de Filología Románica 14 (1): 85-97.

CERRÓN PALOMINO, Á. (2015). "Morphological adjectival intensifier variation in Lima, Perú”, en Hispanic Linguistics at the Crossroads. (Eds. Rachel Klassen et al.). Amsterdam: John Benjamins: 233-251.

DÁSKOVÁ, A. (2006). Sufijos -ada, -ado, -azo, -aje: análisis morfofonológico y semántico, Tesis doctoral, Brno: Masarykova Univerzita.

DE BRUYNE, J. (1979). “Le suffixe -ón en espagnol moderne”, Linguistica antverpiensia 13: 7-53.

FÁBREGAS, A. (2010). “A syntactic account of affix rivalry in nominalizations”, en The syntax of nominalizations across languages and frameworks. (Eds. Artemis Alexiadou y Monika Rathert). Berlin: Mouton: 67-93.

FÁBREGAS, A. (2014). “Argument structure and morphologically underived nouns in Spanish and English”, Lingua 141: 97-120.

FÁBREGAS, A. y MARÍN, R. (2014). Severing eventivity from dynamicity, Manuscrito Inédito, Tromsø-Lille: Universidad de Tromsø / Universidad Lille 3.

FÁBREGAS, A. y VARELA, S. (2006). "Verb classes with eventive infinitives in Spanish”, en Selected Proceedings of the 9th Hispanic Lnguistics Symposium. (Eds. Nuria Sagarra y Almeida J. Toribio). Cascadilla: Somerville: 24-33. 
FAILTELSON-WEISER, S. (1980). Les suffixes quantificateurs de l'espagnol. Paris: Ed. Hispaniques.

GRIMSHAW, J. (1990). Argument Structure. Cambridge (Mass.): MIT Press.

HALE, K. (1986). "Notes on world view and semantic categories: Some Warlpiri examples", en Features and projections. (Eds. P. Muysken y H. van Riemsdijk). Dordrecht: Foris: 233-254.

HALE, K. y KEYSER, S. J. (2002). Prolegomenon to a Theory of Argument Structure. Cambridge (Mass.): MIT Press.

JACKENDOFF, R. (1997). The architecture of the language faculty. Cambridge (Mass.): MIT Press.

LÁZARO MORA, F. (1999). "La derivación apreciativa”, en Gramática descriptiva de la lengua Española. (Dirs. Ignacio Bosque y Violeta Demonte). Madrid: Espasa: 4645-4683.

MARÍN, R y MCNALLY, L. (2011). "Inchoativity, change of state, and telicity: Evidence from Spanish reflexive psychological verbs”, Natural Language and Linguistic Theory 29: 467-502.

MATEU, J. (2002). Argument Structure. Relational Construal at the Syntax-Semantics Interface, Tesis doctoral. Barcelona: Universitat Autònoma de Barcelona.

MILLÁN URDIALES, J. (1978). "Sobre las voces españolas terminadas en -ón”, en Estudios ofrecidos a Emilio Alarcos Llorach, vol. 4. Oviedo: Universidad de Oviedo: 181-208.

MOURELATOS, A. (1978). "Events, processes and states”, Linguistics and Philosophy 2: 415-434.

NGLE (2009). RAE \& ASALE, Nueva gramática de la lengua española. Madrid: Espasa.

OLTRA-MASSUET, I. y CASTROVIEJO, E. (2014). “A syntactic approach to the morpho-semantic variation of -ear", Lingua 151: 120-141.

PIÑÓN, C. (1997). "Achievements in an Event Semantics”, en Proceedings of Semantics and Linguistic Theory 7. (Eds. A. Lawson y E. Cho). Ithaca, NY: CLC Publications: 273-296.

RAMCHAND, G. (2008). Verb Meaning and the Lexicon: A First-Phase Syntax, Cambridge: Cambridge Universtiy Press.

ROSS, J. (1973). "Nouniness", en Three dimensions of linguistic research. (Ed. O. Fujimura). Tokio: TEC Company: 137-257. 